Rev. Latinoam. Psicopat. Fund., São Paulo, 19(4), 630-646, dez.2016

http://dx.doi.org/10.1590/1415-4714.2016v19n4p630.4

\title{
El cuerpo en la cura. Movimientos transferenciales e intricación pulsional*1
}

\author{
Derek Humphreys*2
}

Francois Pommier*3

Objetivo: Intentamos establecer las hipótesis de trabajo del analista confrontado a la irrupción del cuerpo en la cura y que permiten elaborar transferencialmente la violencia pulsional. Procedimientos. La experiencia clínica de los autores con un niño establece las bases teóricas que interrogan el rol del cuerpo. Este es luego analizado transferencialmente en tres situaciones clínicas. Conclusiones. La presencia actual del analista es articulada respecto de su deseo. El cuerpo del analista permite actualizar, en la transferencia, el desplazamiento entre el registro yoico imaginario y el registro simbólico en el que emerge el sujeto.

Palabras-clave: Deseo del analista, transferencia, manifestación corporal, Desintricación pulsional

*' El presente artículo ha sido elaborado a partir de un trabajo presentado por los autores en el taller "intricación pulsional e imagen del cuerpo", $3^{\circ}$ coloquio chileno-francés de psicoanálisis y ciencias sociales, "CUERPO” (Santiago, Facultad de Ciencias Sociales de la Universidad de Chile, Octubre 2014) y ha sido posible gracias a la cooperación científica financiada por el programa ECOS-CONICYT (ECOS-Sud 13H01) de los fondos de investigación de los gobiernos de Francia y Chile. Este fondo de financiamiento a la cooperación científica no introduce conflictos de interés al material presentado.

*2 Aix Marseille Université (Aix Marseille, França)

*3 Université Paris Quest/Nanterre-La Défense (Paris, França) 


\section{ARTIGOS}

El cuerpo tiende a hacerse presente en su disfuncionamiento, en la enfermedad, constituyendo el objeto de reflexión por excelencia de la medicina. El cuerpo no se limita, sin embargo, a su sustrato material sino que da cuenta - en la enfermedad, en sus pasiones, sus sufrimientos - de la relación indisociable y singular al psiquismo. Esta se construye a partir de las experiencias precoces de satisfacción y placer, en presencia de un Nebenmensch (Freud, 1895/1979) suficientemente disponible a la vectorización y el apuntalamiento de la pulsión sobre las funciones corporales (Dejours, 2009) en lo que Winnicott ha llamado handling, holding y object presenting (1971). Sólo en estas condiciones el cuerpo será la materia sobre la que se organiza el campo representacional y la superficie de inscripción y de significación de la experiencia. El cuerpo ocupa, así, un lugar que no es secundario en psicoanálisis, tanto en la enfermedad como en la presentificación de un más acá del lenguaje (David-Ménard, 2014) y la expresión, en la cura, de elementos no simbolizados.

La experiencia clínica nos confronta a veces a la violencia de la descarga pulsional no-intricada. Esta manifestación, proveniente de zonas del cuerpo que carecen de una adecuada representación (Déjours, 2006), da cuenta del complejo proceso de apropiación psíquica del cuerpo y de la necesidad de elaborar teóricamente dicho proceso. Elaboración que excede toda referencia a la relación de objeto: la sexualidad infantil, en su intensidad perversa y polimorfa, busca un placer que es independiente del objeto. En este contexto, nos hemos interesado por el efecto de la presencia del analista - la actualidad del cuerpo del analista y su efecto sobre el trayecto pulsional - en estas situaciones a partir de nuestra propia experiencia clínica. Esperamos así dar cuenta de nuestra hipótesis de trabajo respecto de estos elementos que, sin provenir de la lengua y sin representación, hacen irrupción a través del cuerpo en la cura.

Dada la preponderancia y la inmediatez de la búsqueda de placer y de los efectos de la presencia del otro durante los tres primeros años de vida, discutiremos primeramente el caso de

Rev. Latinoam. Psicopat. Fund., São Paulo, 19(4), 630-646, dez. 2016 
un niño en el que la intricación pulsional pasa por la actualización de la búsqueda de satisfacción. Presentaremos luego las experiencias que nos permiten teorizar los efectos dramáticos de esta desintricación en el adulto y los modos de metabolización de este exceso pulsional en la transferencia. En este acercamiento a las expresiones del cuerpo hemos centrado nuestra atención sobre los movimientos transferenciales, evitando todo lo que se refiere a la esfera médica - que exigiría la consideración de aspectos suplementarios complejos. La elección de una perspectiva clínica determina el carácter fragmentario de esta elaboración pero tiene el mérito de dar cuenta de una praxis que nos permite fijar un campo claro. Los elementos clínicos son aportados respetando la confidencialidad y el anonimato de las personas involucradas.

\section{El cuerpo en juego}

Gruesas lagrimas caen de los ojos de Yanis mientras se ocupa de la pintura que realiza sobre la mesa del taller, sin reacción afectiva aparente. La indiferencia de su gesto y la intensidad de sus lagrimas interrogan la imagen integrada del cuerpo.

Yanis tiene 3 años cuando lo recibo en un taller de expresión. A pesar de su actitud reservada, en dos oportunidades, siguiendo el movimiento de otro niño, Yanis ha golpeado a un tercero en la escuela en un acceso paroxístico de violencia del que había sido difícil extraerlo. Sumado esto a sus dificultades de expresión, le ha sido propuesto este taller.

Yanis se resiste a quitarse el abrigo la primera vez que viene, hasta que su madre le explica que debe partir y que quiere verlo aprovechar el taller. Es habitual que la separación sea difícil en esta primera sesión del año y mi atención es retenida durante una buena parte del tiempo por los niños que manifiestan ruidosamente esta dificultad. Yanis se instala rápidamente en la mesa sin decir palabra y comienza a dibujar. Cuando vuelvo a él, sorprendido por su forma de hacerse imperceptible, veo que se obstina por llenar metódicamente de marrón y luego de negro una hoja de papel, mientras caen lágrimas de sus ojos. Cuando trato de contenerlo hablándole de su dibujo, me pregunta por el baño, que le indico. Como no regresa al cabo de unos minutos, voy al baño: ha llenado el excusado de papel higiénico pero sigue tratando de limpiarse y tira la cadena reiteradas veces. Cuando le propongo que parece 


\section{ARTIGOS}

haber terminado, sube su pantalón y toma un nuevo trozo de papel para con el que frota los bordes del excusado y, cuando le digo que este me parece limpio, frota los muros. El final de la sesión se acerca, salimos a la sala del taller, y me quedo con un sentimiento de inquietud, desconcertado, esperando que esta necesidad de percibir y controlar sus límites pueda organizarse en torno a la materia negruzca y excremencial que dibuja. Efectivamente, la semana siguiente Yanis fabrica cilindros de plastilina marrón que corta con tijeras, antes de recomenzar a llenar de pintura negra una hoja de papel. En su necesidad de cubrir todo el papel de negro, agrega agua sobre su pintura, obteniendo una mancha negruzca indiferenciada. Tal como la primera vez, se ha aferrado a su madre al llegar pero entra y se instala a trabajar y, sin tristeza ni sollozos, gruesas lágrimas caen de sus ojos. Me acerco cuando parece haber terminado para acompañarlo a lavarse las manos. El espejo situado sobre el lavatorio de la sala de taller nos pone a ambos ante sus lágrimas, y digo mi reflejo, que aparece detrás de él, rodeándolo, dice - ¡lloras, estás triste Yanis! Me mira sin decir nada, seca sus lágrimas por primera vez y partimos detrás de un grupo de niños que dibuja sobre el pizarrón. A partir de entonces, Yanis sigue evitando el intercambio con los demás pero sus ojos comienzan a seguir los juegos de los otros niños, como si su deseo se articulase allí donde ve surgir la frustración, escenificarse la envidia, expresarse penas y alegrías. Comienza luego a seguirlos a cierta distancia, tomando los objetos que van quedando abandonados. Se aproxima así al ventanal que da a la calle, donde ve gente pasar. Las lágrimas reaparecen, pero esta vez su tristeza se ancla a lo que articulo sobre la ausencia de su madre y su retorno.

Durante estas primeras exploraciones de la pieza, Yanis introduce su mano empuñada en la boca. Como si, habiendo reconocido una imagen de su propio cuerpo capaz de inscribir la diferencia entre él y su madre, fuese necesario llenar el vacío dejado por esta amputación para hacer surgir la ausencia, la frustración o la pena cuando la tensión aumenta - cuando los juegos escenifican intensos movimientos afectivos. Llenar esta sensación de pérdida inscrita visualmente a través de la oclusión orificial para poder reencontrar una continuidad consigo mismo. Esta continuidad en la inscripción de sus percepciones corporales le permite retomar poco a poco los juguetes abandonados por otros para, a partir del resto de energía del que quedan investidos, inventar sus propias historias. Su mano sale así de la boca para comenzar a reparar una camioneta de juguete en la que hace entrar toda una familia. Me cuenta entonces de sus paseos en camioneta con su padre. Y en su pausa ante el ventanal, ahora habitual, donde ve la gente pasar, se torna hacia el pizarrón 
y dibuja por primera vez una figura: un renacuajo. Winnicott hubiese tal vez visto un Self.

En el trabajo con niños resulta impensable no responder cuando un chico nos estira los brazos o nos pide que leamos un cuento, aún si no sabemos cómo o con qué respondemos. Es sólo a posteriori que puedo pensar que para Yanis su madre no se diferencia aún de su representación del mundo y que la separación provoca en él un desgarro en el que no puede reconocerse solo. Cuando un tercero lo nombra en el espejo, conteniéndolo al momento de iniciar el movimiento de separación, surge una imagen capaz de contener la experiencia, permitiendo también la continuidad del movimiento pulsional. Esta inscripción de la experiencia en torno al cuerpo propio, que llamamos percepción, permite a su vez que Yanis fije límites subjetivantes cuando su imagen es nominalmente reconocida. Antes de eso, sus lágrimas no son más que perdida somática sin correlato afectivo, una amputación.

\section{Catástrofe pulsional}

El objeto no es puro efecto de una sensación: esta debe devenir percepción, que será luego rechazada como elemento exterior (Freud, 1925/1985). La ausencia de un objeto, en su capacidad de vectorizar la sobrecarga pulsional, deja al sujeto a la merced de la búsqueda de satisfacción que caracteriza la sexualidad primordial, impidiendo la adecuada organización del cuerpo erótico (Déjours, 2011). Para poder ir más allá de los problemas de desarrollo y de entorno en esta elaboración, no nos detendremos sobre el objeto sino que desarrollaremos la calidad sexual primordial de la manifestación pulsional y la manera en que esta intensa carga energética impacta al analista en un movimiento caótico, tomando cuerpo en la sesión.

Freud utiliza la metáfora energética al pensar el aparato psíquico como una máquina que almacena energía siguiendo movimientos de carga $\mathrm{y}$ descarga que se asocian al placer/displacer y que integran un sistema complejo de experiencia, memoria y palabra. Esta concepción lo lleva a la formulación de un concepto límite entre psique y soma y arquitecto de la organización orificial, la pulsión. En constante evolución, la pulsión no tiene más que avatares momentáneos. Su capacidad de organización tiene su reverso en su destructividad, patente en las situaciones clínicas marcadas por la ausencia de inscripción del objeto, por su negatividad, situación en la que 


\section{ARTIGOS}

zonas sexuales secundarias son excluidas del intercambio y se manifiestan en movimientos puramente somáticos (Déjours, 2006).

Durante el periodo que precede la organización edipiana y que hemos elegido como entrada a esta problematización, la falta de oposición entre placer y realidad induce una equivalencia entre sujeto y objeto, entre ver/ser-visto, pasivo/activo (Freud, 1919/1973). Este conflicto, determinante de la relación del sujeto a su imagen (Lacan, 1956/1994), será nuestro punto de partida en esta reflexión sobre el momento del encuentro, en la sesión, con el cuerpo del analista, cuando el sujeto se desarticula del trayecto pulsional y de su intrincación de placer/displacer para caer en un goce catastrófico. El cuerpo del analista constituye en este instante la materia sobre la que pueden ser inscritos un trayecto pulsional y una transformación subjetiva. Encuentro que se da en el espacio analítico en la medida en que este ofrece la ilusión de continuidad entre el objeto real y el alucinado gracias a un analista que se mantiene disponible, vivo, capaz de callar la ausencia, de no precipitar la interpretación.

\section{Actualizaciones corporales en la cura}

A partir de nuestra experiencia, hemos elaborado las actualizaciones del cuerpo en la cura como (1) Expresiones del cuerpo fuera de la sesión y que surgen en la cura; (2) Expresiones motrices; (3) Expresiones que circulan a través del cuerpo en la sesión como (a) lo que el analista percibe de la expresión emocional a través del cuerpo del analizante o (b) lo que el analizante percibe de las emociones del analista a través del cuerpo.

Las primeras se refieren a lo que se dice del cuerpo (dicho no es siempre sinónimo de simbolizado). Es el caso de un padre de familia que llamaremos Bastian que, tomado por pulsiones homosexuales intempestivas, se acerca al psicoanálisis temiendo el derrumbe de su universo ante la fuerza de estas pulsiones: el cuerpo le escapa por momentos, arrastrándolo a aventuras puntuales intensas. El vínculo entre estas prácticas secretas y una fantasmatización extremadamente sexualizada se establece rápidamente a través de la presencia omnipotente, en Bastian, de la imagen de su padre. El sueño de un padre que confiesa su atracción por él y en el que se refiere a un intercambio erótico que habría tenido lugar en la realidad, dando un lugar a cada uno en la escena, es interpretado por Bastian como una forma calmar su sentimiento de culpabilidad. Bastian asocia una relación homosexual de juventud a una serie 
de alucinaciones mórbidas. La figura del niño, omnipresente en sus fantasmas hasta el punto de una relación física, aparece en una configuración invertida, maternante, como si el padre fuese una madre o la madre un padre.

No hay instrumentalización ni apoderamiento por reducción del otro al rango de objeto parcial, ni la voluntad de someter al otro que caracteriza la perversión en estas tendencias que podrían parecer perversas; se trata más bien del paradigma perverso al que nos confronta la megalomanía infantil. Bastian juega efectivamente con objetos parciales, haciendo de él mismo un objeto parcial. La progresión de la cura hace aparecer la escisión y hasta una cierta fragmentación entre "por una parte la seducción, por otra la producción" (Baudrillard, 2004). La seducción situada del lado de la forma, de la reversibilidad, del intercambio simbólico, del cuerpo, de la apariencia; la producción del lado de lo obsceno, de lo que se deja ver, de la fractalización.

Estas expresiones son también las de aquellos pacientes que hacen referencia al cuerpo para hablar de un sufrimiento psíquico. Especialmente en pacientes de origen africano o indo-americano, por ejemplo, en los que una cefalea esconde habitualmente una depresión.

Las expresiones emocionales que percibimos a través del cuerpo del analizante, y que asociamos a aquellas que el analizante percibe en su analista, se refieren a los signos físicos de un temor incontrolable o, por el contrario, de gran relajo de algunos pacientes. Estas manifestaciones pueden producir reacciones contra-transferenciales particulares. El llanto, que tiene un estatuto particular en la transferencia cuando está en armonía con un conjunto perceptual, puede a veces ser utilizado como resistencia a la transferencia. La progresión del análisis exige una ruptura que pasa por la posibilidad de anclar esta manifestación de resistencia en lo real. Es el caso de una mujer originaria del norte de África, instalada en Francia desde hace varios años, habiendo dejado a sus hijos (hoy adultos) en su país de origen. A penas llega, comienza sistemáticamente a sollozar, lamentándose de su situación y del destino de estos hijos con los que habla a menudo por teléfono y que no hacen más que acosarla verbalmente exigiendo dinero. Su llanto, que parecía autentico y comprensible durante la primera consulta, se instala como algo inmutable e incoercible hasta el hipo, como el llanto de un niño. Al cabo de 10 sesiones en que el mismo guión de quejas y llanto se repite, no aguanto más la ausencia de elaboración y decido evitar a toda costa estos sollozos que me parecen sin sentido. En una dinámica de odio, sólo pienso en la manera de hacerla dejar de llorar. Lo logro brevemente cuando le digo que me parece que nada cambia en su funcionamiento, ni frente a mí, a sus hijos ni a su soledad. Llega 


\section{ARTIGOS}

sonriente y maquillada a la sesión siguiente y cuando veo que va a comenzar a llorar, interrumpo la sesión brutalmente. Si salgo aquí de la neutralidad, no es para dejarme deformar, informar, por la queja del otro. Lejos de cualquier consideración teórica, aún sabiendo que "la función de la queja es el reconocimiento de un distanciamiento respecto del objeto con la intención de suprimirlo" (Dorey, 1981, p. 130), se trata de un movimiento cercano al paso al acto que surge en un momento de vacilación identitaria en el que, captado por la idea de hacer desaparecer la queja, estoy como bajo control, si entendemos en esta situación de apoderamiento una formación defensiva cuya función es ocultar la falta que se ve develada en el encuentro con el otro. Incapaz de manejar la situación, de identificarme a esta mujer encerrada en un sufrimiento - con el que, al no cederme una parte, me instrumentaliza como superficie de proyección - surge en mí la destructividad de la pulsión de muerte al mismo tiempo que intento salvar algo que me parece ser del órden del deseo cuando me des-identifico a la $10^{\circ}$ sesión, quedando a merced de mi contra-transferencia, a la que adhiero sin reflexión en un primer tiempo para luego decidir seguirla. He sentido ante mí un afecto importante pero no logro encontrar su sujeto.

Ser psicoanalista es poder escindirse constantemente, lo que no logro con esta paciente. Aunque logra desestabilizarme, el trabajo que realizo con ella no tiene ningún efecto y toda intervención mía la hace llorar. "Puedo intentar identificarme al sufrimiento de un paciente deprimido", dice Fédida (2007), “(...) pero para imaginar este sufrimiento tengo que separarme de mi conocimiento del semejante, introduciendo esta diferencia en el semejante (...)" (p. 107), siendo siempre la transformación de las representaciones propias, su deformación, la dificultad mayor. Si intento denominar las pre-representaciones que provienen de la sensorialidad y la motricidad de esta paciente y que constituyen el croquis de un espacio para nuestro intercambio, diría que es una figura de odio.

Algunos pacientes, por último, dan un lugar al cuerpo en la sesión a través de la expresión motriz, ya sea por su inercia o su dinamismo. En torno al diván, algunos insisten en recostarse, otros se resisten a hacerlo. Notamos también cambios bruscos de posición. La manera en que el diván es utilizado debe ser anticipada por el analista ya que a veces, en vez de dar lugar a la fantasmatización, viene a reforzar las defensas o a confortar la sintomatología. El caso de Marcel ilustra el modo en que el dispositivo singular de la situación analítica puede permitir la emergencia del sujeto a través del modo de presencia del cuerpo en la sesión. Marcel, aún joven estudiante cuando 
comienzo a recibirlo hace 15 años, evoca una serie de trastornos que van de la masturbación compulsiva a fantasmas violentos de dominación hacia las mujeres, pasando por la sensación de cambiar constantemente de personaje y de ser objeto de burlas de su entorno. Se siente a menudo en una especie de tensión entre el bien y el mal que no se manifiesta como hostilidad hacia el otro sino por ideas agobiantes, particularmente la de relaciones sexuales múltiples fuera de las que tiene con su pareja. La connotación sexual de estos fantasmas, así como la relación a sus padres, con los que se comporta como un niño, le hacen sentir infantil. Se siente diferente de los demás hasta el punto de dudar de su propia sexualidad, idea que lo avergüenza. La primera parte de su análisis, marcada por la vergüenza y la dificultad de encontrar su lugar entre los otros, oscila entre una adhesión inmediata a la palabra del otro y el rechazo, pasando por movimientos impulsivos irrefrenables que en seguida se reprocha. Como si no supiese a quién dirigir su entusiasmo, Marcel se presenta como un apasionado, no en el sentido psiquiátrico sino el de una "erotomanía originaria fundamental" (Gori, 2002, p. 61), como lo atesta su relación afectiva exclusiva con su pareja y su comprometimiento estable en causas políticas. Su capacidad de asociación y hasta de abandonarse a la fantasmatizacion, que atrapa al vuelo y que trae a la sesión con naturalidad (y con un humor que traduce tanto su incomodidad con esta como su voluntad de comprender el universo conflictual en el que se encuentra enclavado) son impresionantes durante este periodo. Aunque avanza hacia el conocimiento de sí-mismo, tiende a eliminarme: "tengo la impresión a veces que usted soy yo... usted ordena mi pensamiento, organiza el debate, pero en realidad discuto conmigo mismo". Por una parte me hace desaparecer, por otra se fusiona conmigo (no solamente no hay otro, sino que él y yo hacemos una sola persona). Podríamos evocar aquí las palabras Lacan cuando, rechazando el punto de vista de su propio analista, que estimaba que un Yo supuestamente autónomo se apoyaba sobre el YO del analista para hacerse fuerte y sabio, afirma que el YO debe devenir lo que no era, venir ahí donde se sitúa el sujeto. En Marcel el Yo se volatiliza apoyándose en el mío o acaparándolo. Marcel detiene el análisis en torno a una interrogación mutua respecto del interés de su cuestionamiento en un momento de logros sociales y afectivos. Lo retoma 3 años después, cuando su carrera profesional ha avanzado, ha tenido un hijo y su padre ha fallecido. En este preciso momento, la pareja se separa de él, dejando a Marcel ante las mismas angustias y los mismos momentos de excitación incoercibles que vivía antes, a lo que se agrega ahora el odio hacia su ex-pareja. Después de un primer periodo de cólera y quejas depresivas durante 
el cual no logra transformar de manera positiva su destructividad, adopta un nuevo look, sintiéndose de pronto que todo es nuevo. Su cuerpo lo sobrepasa, lo desborda. Habla, en una especie de hipomanía, de la importancia de sentirse bien, de las mujeres que frecuenta. Me dice que al abrirle la puerta le he parecido muy elegante y se pregunta si seré homosexual. Me explica que se reapropia paulatinamente de su cuerpo, que trata de "hacer hablar su cuerpo". El estado hipomaniaco dura un año, hasta que decide hacer el balance de su trabajo, y comprende que en su discurso sobre sus nuevas relaciones ha perdido la traza de su infancia y su adolescencia. La repetición constituye, en este periodo, un intento de reapropiación. Dice sentirse fuerte, en circunstancias que el analista lo siente narcísicamente débil, buscando auto-congratularse permanentemente. Es un momento de refuerzo yoico en el que el Yo de Marcel se aferra a la imagen, inmovilizándolo, y en el que la transferencia es de-narcisizante. Su manera de creer en el objeto le permite rechazar su pérdida. La idea de "deshacerse de una cierta urgencia" aparece finalmente cuando formulo en sesión un estado depresivo subyacente y que permite a Marcel reconocer un sentimiento de soledad. Dice entonces, haciendo ahora coincidir la primera persona, la que habla, con su YO, que estos dos años han sido difíciles y que debe hacer el duelo de la relación con la madre de su hijo. El Yo parece reconstituirse cuando habla en nombre propio y no desde el exterior, como lo hacia durante su estado hipomaniaco. "No sé muy bien como se hizo la transferencia, pero me ayudó el que usted fuese un hombre; usted ha dado cuerpo al burgués, al intelectual, al homosexual....". Me parece que es en este momento que el trabajo analítico comienza, cuando la imagen de sí-mismo se deshace, haciendo emerger un deseo. Como marcando el fin de un ciclo, el trabajo comienza cuando se abre al analista la pregunta por el fin de análisis. Durante el largo periodo hipomaniaco, había sido imposible interrumpir el ciclo en el que se encontraba Marcel, gratificándose narcísicamente en sus relatos de conquista. Mi apuesta fue la de no interrumpirlo, apoyándome en el cuadro, aceptando a veces largos retrasos. El cuadro de la cura se hizo así puro espacio, la geografía primando por sobre la historia.

Marcel decide entonces cambiar de posición. Durante una sesión, se sienta en el diván y me habla de perfil. Resurge en este periodo un elemento de su historia: a los 4 años, jugando con un camarada, sufre un accidente de un ojo que lo lleva, al cabo de 10 años, a perder una buena parte de su capacidad visual. Marcel asocia este accidente a una cirugía de la infancia por fimosis, lo que lo lleva a hablar de una especie de tabú surgido en la pre-adolescencia que reúne el ojo dañado, la sexualidad y un sentimiento de 
vergüenza sexual que durará hasta la edad adulta. Durante el primer periodo de análisis no ha sido posible situar estos dos eventos significativos, como si el analista se encontrase atrapado por la imagen perfecta de la condensación de un complejo de Edipo y de castración, pero reconociendo al mismo tiempo la necesidad de de-construir este magma o de ayudar a Marcel a de-construirlo en la transferencia. Evitar el exceso de sentido para dar lugar a algún sentido. Es solamente cuando la hipomanía comienza a mermar y que Marcel habla de este ojo investido de modo ambivalente, integrando su hándicap a su existencia, que decido hablar de la posibilidad de una intervención sobre su ojo. En lugar de poner el acento sobre el vínculo directo entre el ojo y la sexualidad, con el exceso de sentido que esto arrastraría, intento así abrir la pista no de una recuperación de su visión, sino de su duda permanente sobre la asociación entre esta incapacidad que se agrava y que dice hacerse notoria y su dificultad para seducir una mujer.

\section{Conclusión}

Hemos intentado apuntalar teóricamente algunos momentos claves de la clínica en los que el analista se ve confrontado a la violencia de la desintricación pulsional. Esta elaboración de los efectos de la inadecuada apropiación psíquica del cuerpo nos ha permitido interesarnos por la presencia del analista en lo que llamaremos primeramente un "espesor de presencia", sensible en una cierta capacidad de responder, de pensar, de ser afectado, antes de analizar sus efectos transferenciales.

El trabajo con un niño pequeño nos ha permitido ilustrar la importancia actual del cuerpo del analista en un registro que antecede la separación entre Yo/No-yo y en el que tampoco cabe una distinción entre objeto y placer. Apoyándonos sobre el principio de un going on being (Winnicott, 1954) como continuidad del que "esta siendo y estando", proponemos que en el encuentro con otro es posible conciliar la representación y la experiencia corporal en la constitución de una especie de tejido de continuidad (dependiente inicialmente de la presencia del que aporta un objeto y que es también capaz de reconocer los efectos de esta introducción). Hemos reconocido en este análisis la posibilidad de establecer un borde en el encuentro: el aparato para pensar del analista se manifiesta en la capacidad de reconocer e investir el cuerpo del niño como separado, individual, habitado por sus propias sensaciones. 


\section{ARTIGOS}

En el espejo, Yanis se reconoce separado, individual, cuando la imagen fija un límite entre él y el otro. A partir del establecimiento de esta primera separación que funda una singularidad y un límite corporal que lo diferencia del entorno, Yanis organiza una superficie de separación y de contacto que permite tanto la apropiación de la sensación como la posibilidad de contener estas sensaciones, pensamientos y sentimientos en la representación de sí en el espejo: una percepción de sí-mismo. Un tercer tiempo se organiza en torno a las cavidades y los orificios cuando Yanis descubre el espacio vacío de las cavidades de su cuerpo, que comienza a llenar de representaciones cuando la tensión pulsional resulta desbordante, cuando el vacío de la ausencia da acceso a la profundidad angustiosa del tiempo y del espacio (Kahn, 2012). Lejos de la normatividad edipiana, hemos referido esta exploración de los niveles de organización del cuerpo a los organizadores psíquicos capaces de hacer emerger una simbolización ante la ausencia (Baruch, 2011).

Hemos referido el encuentro capaz de favorecer el proceso de re-intricación, en un primer tiempo, a los procesos afectivos y de pensamiento del analista, que dependen de elementos contingentes (David-Ménard, 2011) en los que el analista es "sorprendido". Pensamos que el establecimiento de una fiabilidad de la presencia del otro como fundamento de la capacidad de ilusión depende del juego en el que nos dejamos sorprender. Este modo de presencia física del que responde al llamado de un niño ha sido el fundamento de nuestra elaboración del lugar del cuerpo (de analista y analizante) en la cura con adultos en situaciones en las que opera más la presencia que la función del analista. La posibilidad de ver al analizante, de anclarlo visualmente cuando este va hacia un naufragio en el diván, resulta organizador ante estas experiencias. El reconocimiento de una imagen especularizable permite la apropiación actualizada de una continuidad pulsional que da acceso a la a la figuración (Kahn, 2012) y a la fantasmatización.

Pensamos que el deseo del analista esta implicado en esta afirmación de la presencia y la disponibilidad del analista que no hemos llamado aún transferencia. Tanto en nuestra respuesta al llamado de un niño como en nuestra posibilidad de llamar y acoger al niño que se manifiesta en la desintricación del adulto y al "niño en el analista" que se deja sorprender (Guignard, 1994).

El momento en el que Marcel accede al universo simbólico ilustra el movimiento transferencial que permite que el deseo venga finalmente a ocupar el lugar del Yo. Sin osar sugerir que la afirmación subjetiva haya triunfado aquí sobre el Yo, el periodo de hipomanía permitió la lenta reconstitución de la superficie corporal a partir de la que pudo, poco a poco, recuperar 
algunas las trazas con las que se reconstruyó un cuerpo capaz de contenerlas, tal como Yanis hizo entre el reconocimiento en el espejo y la exploración de un interior capaz de contener. Resulta sorprendente la correspondencia entre la constitución de una superficie y el acceso a la profundidad, que permite que Marcel se diferencie del analista en la reconstrucción de su propio Yo. El deseo del analista, "concepto regulador" y "respuesta al enigma del deseo" (Guyomard, 2011), resulta fundamental al proceso de transformación del analizante, entre el Yo como constructo imaginario y la afirmación subjetiva, y luego entre el sujeto que (se) enuncia y un Yo unificado. Haciendo de la situación analítica el dispositivo singular de emergencia del sujeto en el desplazamiento transferencial, a través del cuerpo en la cura, entre el registro yoico imaginario y el registro simbólico. El deseo del analista manifestado en su presencia parece, en suma, interpelar otro deseo, de re-encontrarse consigo mismo para devenir un sujeto que prevalece por sobre la dependencia y la deuda respecto del objeto.

Agradecimientos: Agradecemos al fondo ECOS-CONICYT, que ha hecho posible un trabajo conjunto regular en la elaboración de esta reflexión. Agradecemos también la invitación a trabajar formulada por el Laboratorio de Psicoanálisis y Practicas Sociales (LaPSoS) de la Universidad de Chile y a presentar el primer esbozo de este trabajo conjunto en el coloquio FrancoChileno "CUERPO" organizado por la Facultad de Ciencias Sociales de la Universidad de Chile en Octubre del 2014. Agradecemos también la generosa ayuda de Li Feng y de Beatriz Santos, cuya lectura experta nos ha permitido establecer un resumen adecuado en mandarín y en portugués.

\section{Referencias}

Baruch, C. (ed.) (2011). Nouveaux développements en psychanalyse. Autour de la pensé de M. de M'Uzan. Sèvres: Éditions EDK.

Baudrillard, J. (2004). Mots de passe. Paris: Biblio essais.

David-Ménard, M. (2011). Eloge des hasards dans la vie sexuelle. Paris: Hermann.

David-Ménard, M. (2014). Corps et langage en psychanalyse. Paris: Campagne première.

Déjours, Ch. (2006). Le rêve: révélateur ou architecte de l'inconscient? Psychiatrie française, 36, 7-38. 


\section{ARTIGOS}

Déjours, Ch. (2009). Corps et psychanalyse. L'information psychiatrique, 85(3), 227-234 .

Déjours, Ch. (2011). Le corps entre "courant tendre" et "courant sensuel". Revue Française de psychosomatique, 40 (2), 21-42.

De M'Uzan, M. Reconsiderations et nouveaux développements en psychanalyse. In C. Baruch (Ed.) (2011), Nouveaux développements en psychanalyse. Autour de la pensé de M. de M'Uzan (pp 19-31). Sèvres: Éditions EDK.

Dorey, R. (1981). La relation d'emprise. Nouvelle revue de psychanalyse, 24, 117140.

Fédida, P. Humain/Deshumain. In P. Fédida et al. (2007), Humain/Deshumain. Pierre Fédida, la parole à l'œuvre (pp. 11-126). Paris: PUF.

Freud, S. (1985). La négation. In Résultats, idées, problèmes II (pp. 135-139, trad. fr. J. Laplanche). Paris: PUF. (Trabajo original publicado en 1925).

Freud, S. (1973). "Un enfant est battu": contribution à la connaissance de la genèse des perversions sexuelles. In Névrose, psychose et perversion (pp. 209-218, trad. fr. D. Guérineau). Paris: PUF. (Trabajo original publicado en 1919).

Freud, S. (1979). L'esquisse d'une psychologie scientifique. In La naissance de la psychanalyse (pp. 307-396, trad. fr. A. Berman). Paris: PUF. (Trabajo original publicado en 1895).

Gori, R. (2002). Logique des passions. Paris: Denoël.

Guignard, F. (1994). L'enfant dans le psychanalyste. Revue Française de Psychanalyse, 58(3), 649-659.

Guyomard, P. Lacan et le contre-transfert: le contrecoup du transfert. In P. Guyomard, et cols. (Ed.) (2011), Lacan et le contre-transfert (pp. 11-91). Paris: PUF.

Kahn, L. (2012). L'écoute de l'analyste. De l'acte à la forme. Paris: PUF, Fil rouge.

Lacan, J. (1994). Le séminaire. Livre 4. La relation d'objet. Paris: Seuil (Trabajo original publicado en 1956).

Winnicott, D.W. (1971). Playing and reality. New York: Routhledge.

Winnicott, D.W. (1954). Mind and its relation to the psyche-soma. British Journal of Medical Psychology, 27(4), 201-209.

\section{Resumos}

(O corpo na cura. Movimentos Transferenciais e combinação pulsional)

Objetivo. Estabelecemos hipóteses relativas ao trabalho do analista que enfrenta a irrupção do corpo no tratamento que permitem elaborar na transferência 
a violência da pulsão. Procedimentos. A experiência clínica dos autores com uma criança pequena constitui a base teórica a partir da qual o papel do corpo é questionado. As atualizações do corpo no tratamento são então analisadas em sua relação à transferência em 3 três situações clínicas. Conclusões. A presença atual do analista é articulada ao seu desejo. O corpo do analista abre à atualização, na transferência, do deslocamento entre o Eu imaginário e Eu simbólico no qual surge o sujeito.

Palavras-chave: Desejo do Analista, transferência, manifestação do corpo, desfusão pulsional

(The body in cure. Transferential movements and drive combination)

Purpose: We establish hypotheses referring to the work of psychoanalysts' who are confronted by the drive violence that characterizes the sudden emergence of the body within de psychoanalytical setting. Procedures: The clinical experience of the authors with a small child provides the theoretical basis for analysis of the role of the body. Three different clinical situations are then transferentially analyzed. Conclusions: The presence of the psychoanalyst is linked to his desire. The body of the psychoanalyst allows is open to actualization, in transference, of displacement from an imaginary Self towards a symbolic Self in which the subject emerges.

Key words: Desire of the analyst, transference, manifestation of the body, drive desintrication

(Le corps dans la guérison. Mouvements transférentiels et intrication pulsionnelle)

Objectif: Nous établissons des hypothèses sur le travail de l'analyste confronté à l'irruption du corps dans le traitement lui permettant d'élaborer transférentiellement la violence pulsionnelle. Procédure: L'expérience clinique des auteurs avec un enfant en bas âge a permis d'établir les bases théoriques à partir desquelles le rôle $d u$ corps est interrogé. Les actualisations du corps dans le traitement sont ensuite illustrées à travers trois situations cliniques. Conclusions: La présence actuelle de l'analyste est référée à son désir. Le corps de l'analyste ouvre à l'actualisation, dans le transfert, du déplacement du moi en je, puis du je vers un moi unifié.

Mots clés: Désir de l'analyste, transfert, manifestation corporelle, déliaison pulsionnelle

(Körper und Heilung. Transferenzielle Gebärden und Triebkombination)

Ziel. Es sollen Arbeitshypothesen für die Arbeit des Analytikers formuliert werden, der mit der plötzlichen Manifestation des Körpers während der Heilung konfrontiert wird, wobei die Triebgewalt in der Übertragung erarbeitet werden kann. Vorgehen. Basierend auf der klinischen Erfahrung der Autoren mit einem Kleinkind werden theoretische Grundlagen entwickelt, um die Rolle des Körpers zu erläutern. 


\section{ARTIGOS}

Die Aktualisierungen des Körpers während der Heilung werden dann anhand dreier klinischer Situationen illustriert. Schlussfolgerungen. Die aktuelle Präsenz des Analytikers ist mit seinem eigenen Begehren verbunden. Der Körper des Analytikers ermöglicht die Aktualisierung, in der Übertragung, der Verschiebung von einem imaginären Ich hin zu einem symbolischen Ich, was zur Manifestation des Subjekts führt.

Schlüsselwörter: Begehren des Analytikers, Übertragung, Manifestation des Körpers, Triebentbindung

(身體癒合。轉讓的動作和組合pulsional)

目的分析家面对治疗中身体的呈现, 这允许他以移情的方式转化冲动 性的暴力, 我们在本文中建立了这一分析家工作的假设。

过程作者和一个低龄儿童工作的临床经历构成了反思身体在治疗中的 作用的基础。在本文中，治疗中身体的呈现通过三个临床片段加以描述。

结论 分析家的在场关联于分析家的欲望。分析家的身体，通过移情的 过程，使得患者由想象的自我朝向符号的自我的转变得以可能，在此过程中主 体得以出现。

关键词 分析家的欲望, 移情, 身体的呈现, 冲动的爆发

Citação/Citation: Humphreys, D., Pommier, F. (2016, dezembro). El cuerpo en la cura. Movimientos transferenciales e intricación pulsional. Revista Latinoamericana de Psicopatologia Fundamental, 19(4), 630-646.

Editores do artigo/Editors: Profa. Dra. Ana Maria Rudge e Profa. Dra. Sonia Leite

Recebido/Received: 11.9.2016/ 9.11.2016 Aceito/Accepted: 15.10.2016 / 10.15.2016

Copyright: (C) 2009 Associação Universitária de Pesquisa em Psicopatologia Fundamental/ University Association for Research in Fundamental Psychopathology. Este é um artigo de livre acesso, que permite uso irrestrito, distribuição e reprodução em qualquer meio, desde que o autor e a fonte sejam citados / This is an open-access article, which permits unrestricted use, distribution, and reproduction in any medium, provided the original authors and sources are credited.

Financiamento/Funding: Pesquisa financiada pelo ECOS-CONICYT / Research funded 


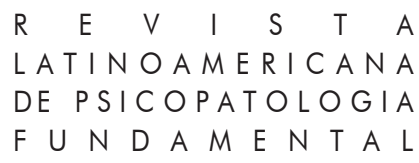

by ECOS-CONICYT.

Conflito de interesses/Conflict of interest: Os autores declaram que não há conflito de interesses / The authors have no conflict of interest to declare.

\section{DereK HuMphreys}

Médico; Doctor en Psicopatología Fundamental y Psicoanálisis; Psicoanalista; Maître de Conférences, Aix Marseille Université, LPCLS EA 3278 (Aix-en-Provence, France).

646 Maison de la Recherche

29 Av. Robert Schuman

13621 Aix-en-Provence cedex 1, France

derek.humphreysmanterola@univ-amu.fr

\section{François Pommier}

Psiquiatra y Psicoanalista; Doctor en Psicopatología Fundamental y Psicoanálisis; Profesor titular, Laboratoire CLIPSYD EA 4430, Université Paris Ouest/Nanterre-La Défense (Paris, France).

61 rue de Lancry

75010 Paris, France

fp@pommier.tel

This is an open-access article, which permits unrestricted use, distribution, the original authors and sources are credited. 